\title{
AUTOTRANSPLANTE DENTAL: CASO CLÍNICO
}

\section{Dental Autotransplantion: Case Report}

\author{
Morales-Bravo Byron Roberto*1 ${ }^{1}$,Cordero-López María Augusta ${ }^{1}$, Vintimilla-Coronel Santiago \\ Efraín $^{1}$,Pinos-Narváez Patricia Alexandra ${ }^{1}$ \\ ${ }^{1}$ Docente de la Carrera de Odontología, Universidad Católica de Cuenca-Ecuador. \\ *byronmorales@yahoo.com
}

\begin{abstract}
Resumen
En este caso clínico se describe el protocolo del autotransplante dental de una paciente de 14 años de edad, que presentaba apiñamiento dental, pieza incluida, caries profundas y extensas e higiene bucal defectuosa. La paciente necesitaba tratamiento de ortodoncia, exodoncias, y cirugía para extraer una pieza incluida. Dentro del plan de tratamiento se decidió realizar la exodoncia de la pieza 2.4 debido a que presentaba una extensa y profunda lesión cariosa, la cual hubiera requerido de un tratamiento de conducto, y probablemente la colocación de un poste y la cementación de una corona o una incrustación, dependiendo de la pérdida de tejido dentario. Se realizó la exodoncia de las piezas 2.4 y 3.5, por razones terapéuticas en el tratamiento de ortodoncia; también se hizo cirugía de la pieza 2.5 la cual se presentaba incluida y palatinizada, además de poseer una raíz corta. Por tener una raíz más larga y además de forma anatómicamente similar a la 2.4, se decidió autotransplantar la pieza 3.5 a este alveolo siguiendo los criterios de selección adecuados, así como una técnica quirúrgica minuciosa dando una alternativa terapéutica ideal al paciente. De esta forma brindamos a la paciente la posibilidad de disfrutar de unos dientes sanos, para continuar el tratamiento de ortodoncia. En el control a los treinta días se observó estabilidad de la pieza 3.5 en el alvéolo de la 2.4; con salud periodontal óptima. Al completar el año comprobamos que la pieza autotransplantada se encontraba con movilidad fisiológica, la encía estaba sana, con valores normales a las pruebas de sandaje periodontal y de sensibilidad pulpar, finalmente la línea media se había corregido.
\end{abstract}

Palabras clave: Diente no Erupcionado, caries, reimplantación.

\begin{abstract}
In this clinical case study we describe the protocol for the dental autotransplantion of a 14-year-old patient, showing dental crowding, an included thoot, extensive cavities and defective oral hygiene. The patient needed orthodontic treatment, extractions and surgery to remove an included piece. The treatment plan,inclued extraction of we extracted piece 2.4 because it showed an extensive and deep carious lesion, which otherwise would had required an endodontic treatment followed by placement of a post and a core crown or an incrustation depending of tissue loss tooth. Piece 3.5 was also extracted for therapeutic reasons. Additionally, piece 2.5 was subjected to surgery because it was included and palatinized, besides having a short root. Due to its longer root and a similar anatomy to piece 2.4,3.5, was autotransplanted into the corresponding alveolus, following a meticulous surgical technique. By doing so, we provided an ideal therapeutic alternative to the patient, leaving a healthy tooth in place to continue with the orthodontic treatment. After 30 days' post-surgery, piece 3.5 was stably positioned in the alveolus of piece 2.4, and exhibited optimal periodontal health. After one year, the autotansplanted piece showed physiological mobility, the gum was healthy, showing normal depths and positive response to pulp sensitivity tests. Finally, the patient's midline has been corrected.
\end{abstract}

Key words: Tooth unerupted, cavities, reimplantation.

\section{INTRODUCCIÓN}

El autotrasplante se refiere a la transferencia quirúrgica de un diente de una posición a otra en el mismo individuo dentro de los alveolos de dientes extraídos o sitios preparados quirúrgicamente. ${ }^{1-4}$ Por lo regular el diente trasplantado es un órgano no erupcionado, parcialmente erupcionado ${ }^{5}$ o erupcionado que puede estar radicularmente inmaduro o maduro. ${ }^{6}$ Para preservar las células del ligamento periodontal y garantizar así el éxito del autotrasplante, el suministro sanguíneo, la nutrición celular y el óptimo contacto entre sus estructuras son fundamentales. Es decir, el factor más importante es la vitalidad del ligamento periodontal del diente trasplantado. ${ }^{5}$ Algunos autores mencionan que el tiempo óptimo para el éxito del autotrasplante es cuando el desarrollo radicular ha alcanzado dos tercios o tres cuartos de la longitud radicular. El abordaje puede ser inmediato o diferido, el primero cuando el sitio receptor está libre de proceso patológico y el segundo cuando se observa un proceso infeccioso el cual 
debe ser eliminado antes del acto quirúrgico. ${ }^{7}$ El autotrasplante ha demostrado ser un procedimiento útil para reemplazar dientes con caries extensas, no restaurables, ${ }^{8}$ dientes fracturados ${ }^{9-12}$ zonas edéntulas por dientes retenidos ${ }^{13}$ agenesia, ${ }^{9}, 11,12$ erupción ectópica, enfermedad periodontal y fracaso endodóncico ${ }^{5,9}$ siempre y cuando un diente donador esté disponible. ${ }^{5,14} \mathrm{El}$ uso de antibióticos en el tratamiento de autotrasplante es necesario sólo cuando no se han erradicado satisfactoriamente problemas locales antes del procedimiento quirúrgico o en pacientes con determinados problemas sistémicos. ${ }^{7}$ El propósito de la descripción de este caso clínico es presentar el procedimiento, y las nuevas alternativas de tratamiento como, por ejemplo, el autotransplante de un diente proveniente de otra arcada del mismo paciente de forma inmediata, en el cual el lecho receptor tenía la anatomía y condiciones adecuadas para recibir el diente.

\section{PRESENTACIÓN DEL CASO CLÍNICO}

Paciente de sexo femenino, de 14 años de edad, que asistió a la consulta presentando dolor dental y manifestó que sus dientes estaban torcidos.

Clínicamente en la inspección intraoral observamos ausencia de la pieza dental 2.5 , caries profunda y extensa de la pieza 2.4 con afección de la pulpa dentaria y desviación de la línea media.(Fig:1;2;3). Una vez realizados todos los elementos de diagnóstico (historia clínica, radiografías, modelos preliminares y fotografías), se le explicó la situación a la paciente en presencia de su representante legal y se elaboró la propuesta del plan de tratamiento. El mismo que constó de la exodoncia de las piezas $2.4 ; 1.4 ; 4.5$; así como de la pieza 3.5 por necesidad en la terapéutica ortodóntica.

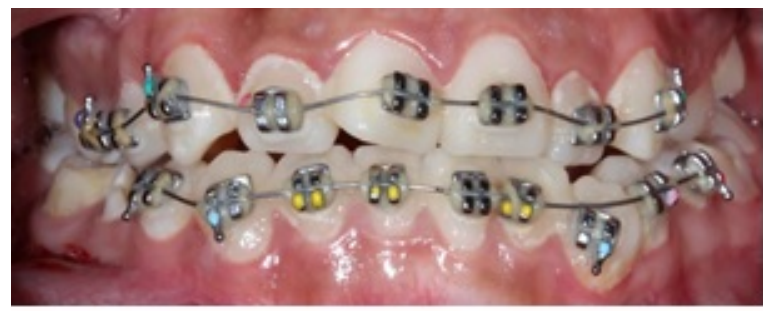

Fig. 1. Línea media desviada

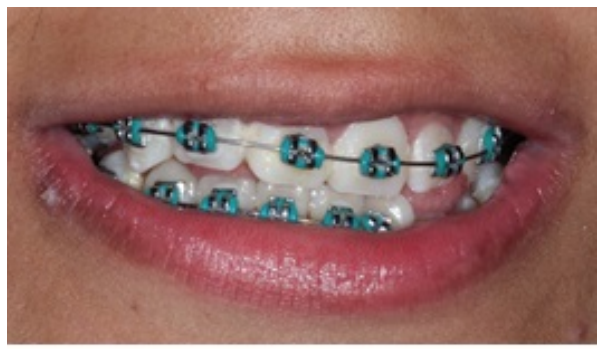

Fig. 2. Línea media desviada

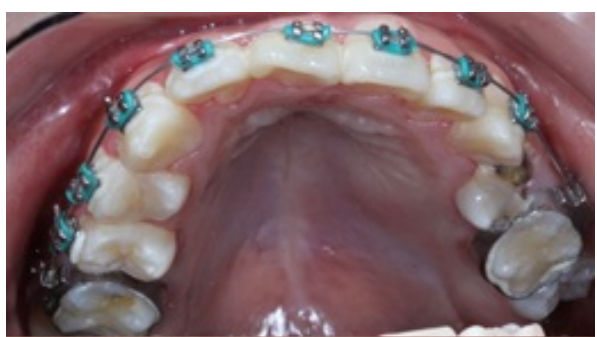

Fig. 3. Caries en pieza dentaria 2.4, ausencia de la pieza dentaria 2.5 .

En la radiografía panorámica la pieza 3.4 presentaba una curvatura en su ápice (Fig:4), condición que no era apropiada para su autotransplante; por lo tanto, se decidió realizar la exodoncia de la pieza 3.5 que presentaba una raíz recta y cónica muy similar al diente 2.4(Fig:11,12), el cual sería extraído por caries extensa que compromete al órgano pulpar. Luego de ser explicado el procedimiento y aceptado por la paciente y su representante, se procedió a la ejecución del mismo.

\subsection{Ejecución del plan de tratamiento}

Como en muchos procesos ortodóncicos, fue necesario extraer ciertas piezas dentales con el fin de lograr el espacio para corregir ciertas anomalías, tanto oclusales como armónicas y estéticas. Es por ello que procedimos a anestesiar y a realizar la extracción simple de las piezas 1.4 y 4.5 , debido a falta de espacio y corrección de línea media. Quince días después realizamos la exodoncia de la pieza 2.4 (Fig:5,6) y la extracción quirúrgica de la pieza 2.5 , levantamiento de colgajo, y su posterior osteotomía, para encontrar la pieza incluida, teniendo los cuidados y respetando tiempos adecuados para no lesionar el alveolo vecino, que sería el lecho receptor de la pieza a autotransplantar.(Fig:7,8)

A continuación, realizamos la extracción de la pieza 3.5 con los cuidados adecuados para evitar traumatismos y lesiones, con el fin de reimplantarla en el alveolo 2.4(Fig:9,10). La pieza 3.5 fue transferida de forma inmediata al alveolo receptor previa copiosa irrigación del alveolo y diente con suero fisiológico, retirando cuidadosamente el coagulo superficial del alveolo, evitando curetear o manipular la raíz(Fig: 13). Insertamos luego el diente en su lecho receptor, cerciorándonos de lograr un óptimo ajuste mediante presión digital suave, y dejándolo en infraoclusión para evitar movimientos del mismo dentro del alveolo. Esto pretendía reducir los movimientos que pudiesen interferir en su posterior oseointegración, revascularización y formación de ligamento periodontal. Una vez hecha la sutura, verificamos la adecuada posición de la pieza en infraoclusión.(Fig:14)

Luego de la cirugía recomendamos reposo y dieta blanda a la paciente; además prescribimos antibióticos y antiinflamatorios por 7 días, con el fin de disminuir la inflamación inicial sobre la membrana periodontal. Ocho 


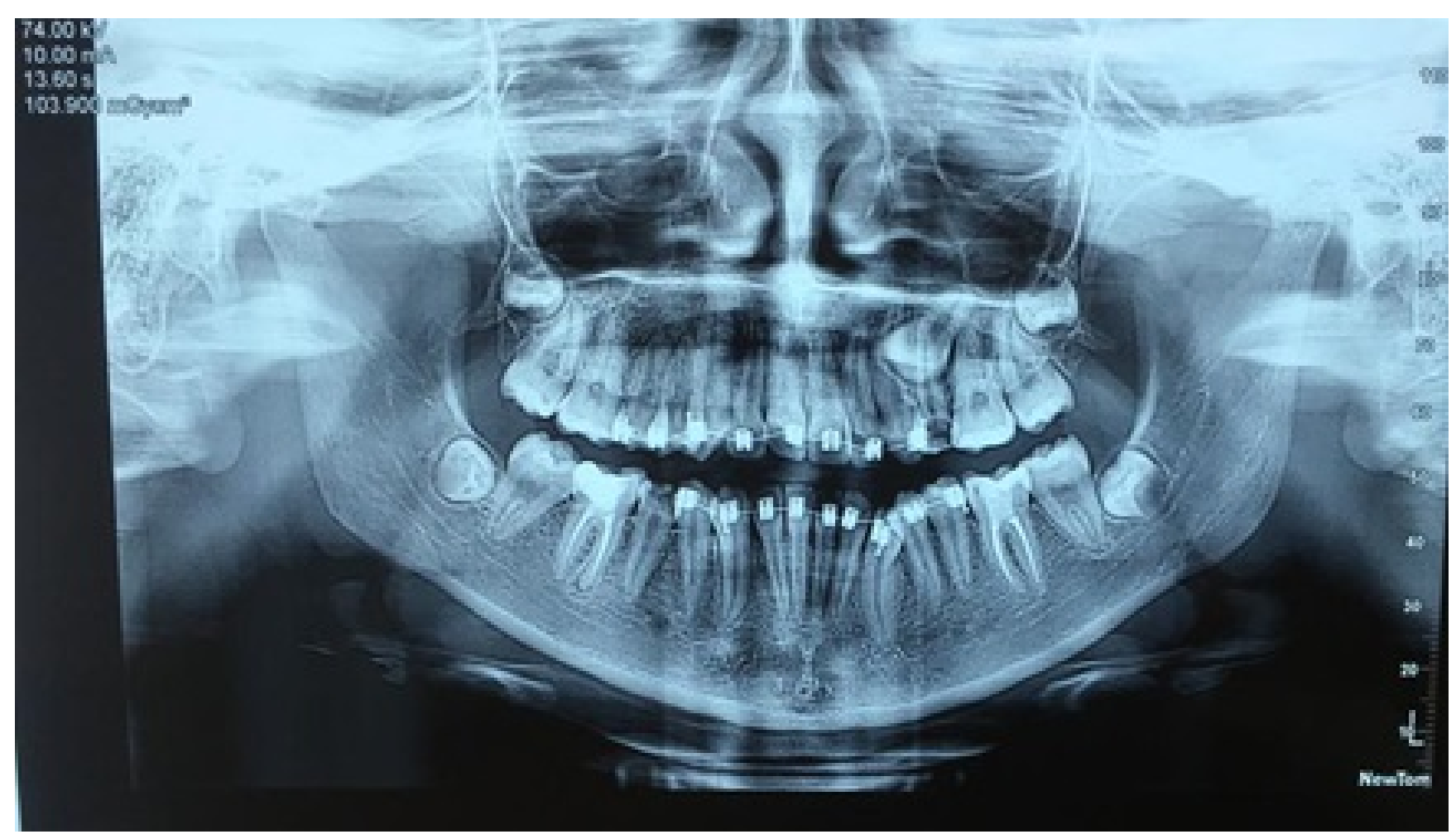

Fig. 4. Radiografía Inicial

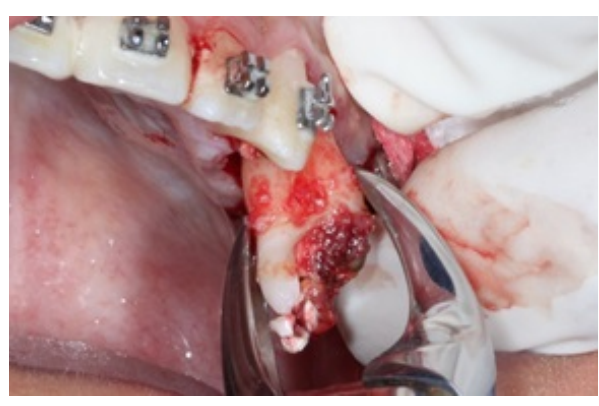

Fig. 5. Extracción simple de la pieza No. 2.4

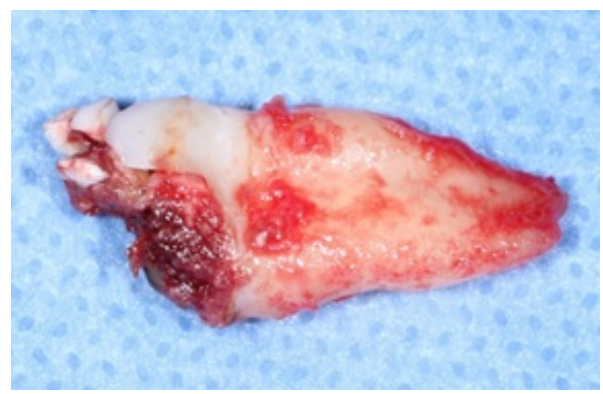

Fig. 6. Extracción simple de la pieza No. 2.4

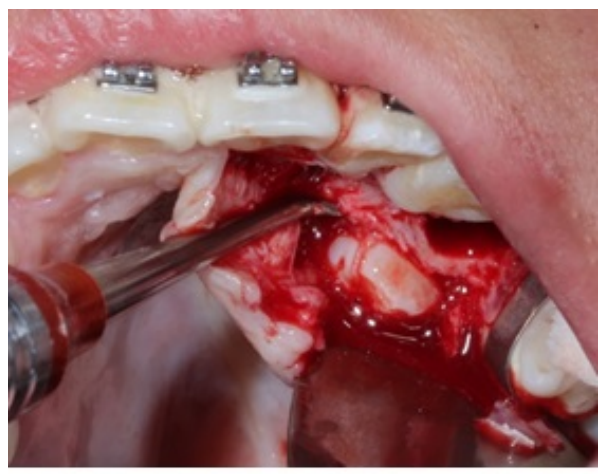

Fig. 7. Extracción de la pieza No. 2.5 incluida

días después, realizamos un control clínico y retiramos los puntos. Un mes después la paciente acudió a una cita de control y nos informó que no existía sintomatología dolorosa o alguna molestia fuera de lo normal; además en esta sesión clínica realizamos un control ortodóntico y la activación de la aparatología.

En los controles trimestrales y al completar el año comprobamos que la pieza autotransplantada se encontraba con movilidad fisiológica, la encía estaba sana, con profundidades al sondeo normales y respuesta normal a las pruebas de 


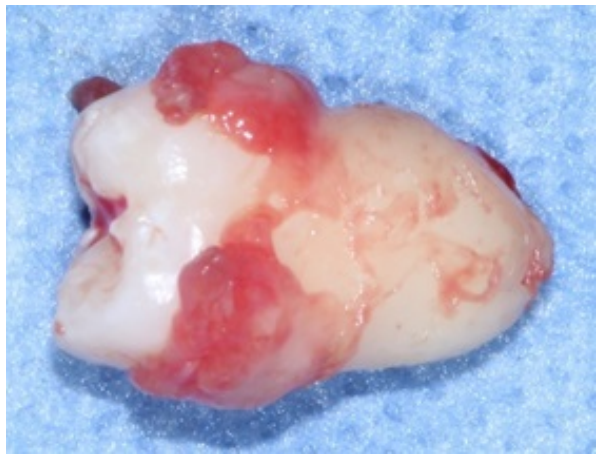

Fig. 8. Extracción de la pieza No. 2.5 incluida

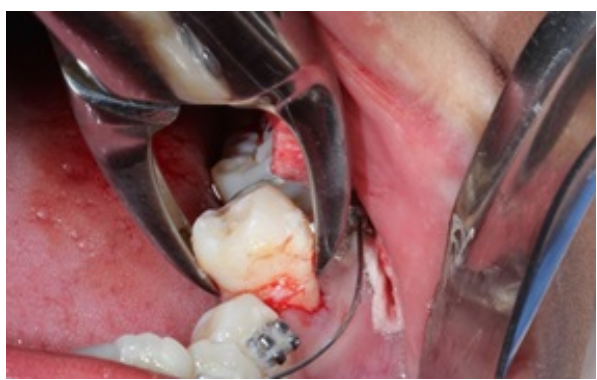

Fig. 9. Extracción de la pieza No. 3.5

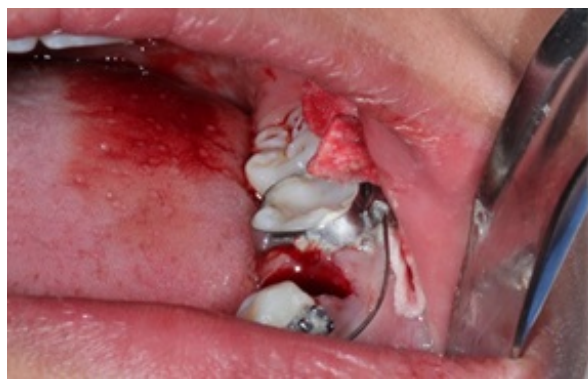

Fig. 10. Extracción de la pieza No. 3.5

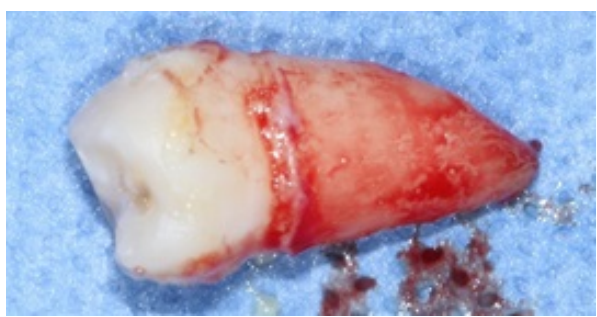

Fig. 11. Raíz cónica y recta similares de la pieza No. 3.5 y 2.4

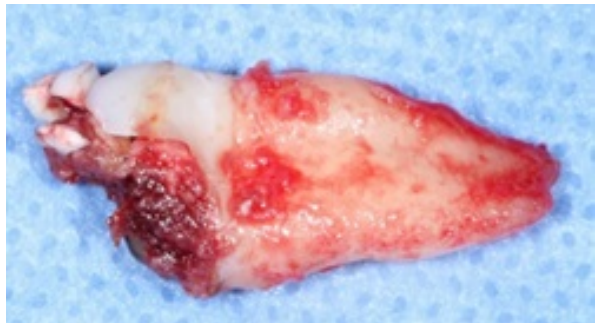

Fig. 12. Raíz cónica y recta similares de la pieza No. 3.5 y 2.4

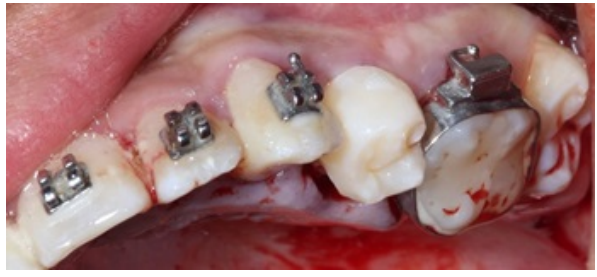

Fig. 13. Autotransplante de la pieza No. 35 en el alveolo receptor correspondiente a la pieza No. 2.4

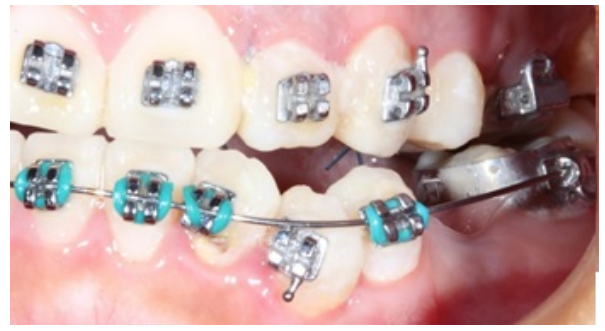

Fig. 14. Sutura y se deja en infraoclusión para evitar movimientos dentro del alveolo

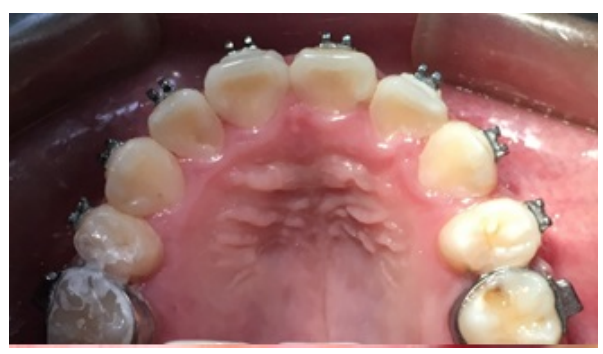

Fig. 15. Pieza autotransplantada al año con encía sana

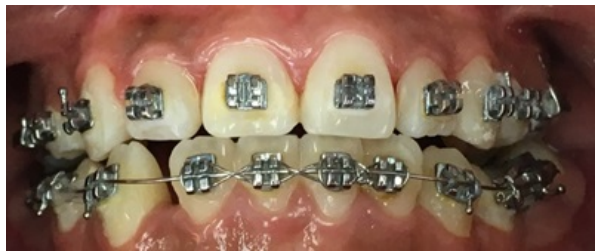

Fig. 16. Línea media corregida 
sensibilidad pulpar y, finalmente, que la línea media se había corregido.(Fig:15;16)

\section{DISCUSIÓN:}

La decisión de esta estrategia terapéutica fue tomada en base a las características presentadas por el paciente tal como zona y pieza de remplazo aptos para el autotrasplante, en este caso en particular el requerimiento de tratamiento ortodóntico permitió acceder a este plan de tratamiento, debido a que la pieza extraída se presentaba con caries extensa y requería tratamiento de conducto, colocación de poste y corona, por lo que el pronóstico de esta pieza no era favorable y posiblemente a largo plazo terminaría en exodoncia, no se procedió a bajar la pieza 25 que se encontraba incluida ya que en el acto quirúrgico al liberar el premolar la posición estaba muy horizontal y se dificultaba la tracción hacia el plano oclusal alargando el tiempo de tratamiento, aumentado la posibilidad de reabsorción radicular y afectado la estabilidad de la pieza en boca por lo que se decidió autotransplantar la pieza que requería extracción por terapéutica de ortodoncia. Algunos autores recomiendan que las piezas dentarias autotransplantadas ${ }^{15-18}$ pueden recibir tratamientos estéticos y ortodóncicos, luego de esperar alrededor de 4 meses ${ }^{19,20}$ siempre y cuando el canal radicular no haya sido obliterado; caso contrario, se debe esperar que esto se dé para realizar la activación de la aparatología ortodoncica. ${ }^{21,22}$ Por el contrario, en el presente caso fue posible realizar los tratamientos respectivos en menor tiempo, basándonos en la evidencia científica de otros casos similares, ${ }^{23,24}$ debido a que los factores circundantes así lo permitieron. Pese a que cualquier diente permanente puede ser trasplantado (siendo de suma importancia la etapa de desarrollo en la que se encuentran ${ }^{15-18}$ ), los premolares son los dientes que con mayor frecuencia son autotransplantados por motivos de ortodoncia. ${ }^{25}$ Una preparación adecuada del sitio receptor y la protección de las células del daño periodontal aumenta la tasa de éxito del autotrasplante, ${ }^{23,24}$ al igual que la revascularización para evitar inflamación y por ende la eficacia del autotransplante dental. ${ }^{9}$ Los controles deben realizarse de forma trimestral durante los 12 primeros meses y anualmente hasta el tercer año luego de la intervención, tal como se reportó en el presente caso durante el primer año. En este caso clínico la pieza autotransplantada presentaba los ápices inmaduros, lo que favoreció a la pronta estabilización del ligamento periodontal y la revascularización pulpar, mejorando el pronóstico inicial del tratamiento y su posterior evolución. Es importante mencionar que cuando la pieza donante está madura, la regeneración vascular y en general la estabilización periodontal se ven afectadas debido a que no permite un favorable crecimiento óseo alveolar. Además, se recomienda realizar el tratamiento de conducto para ampliar los criterios de éxito de la pieza autotransplantada. ${ }^{19}$

\section{CONCLUSIONES}

El objetivo del autotransplante dental es remplazar aquellas piezas dentarias que se encuentran en mal estado El autotrasplante se considera una alternativa terapéutica menos agresiva y más económica que los implantes dentales, además de evitar tratamientos más agresivos como prótesis e implantes dentales. Esto es así, siempre que existan las condiciones adecuadas en el sitio receptor y el diente donador, lo cual permite aumentar la probabilidad de éxito, el cual también depende del estadío de desarrollo y de la protección de las células periodontales. La reimplantación y el autotransplante constituyen una buena decisión terapéutica cuando el paciente no tiene nada que perder y, por el contrario, gana la permanencia de un diente funcional siempre y cuando las condiciones sean óptimas.

Agradecimiento: Los autores agradecen al Dr. Luis Andrés Yarzábal (Departamento de Investigación, Carrera de Odontología. Universidad Católica de Cuenca) por su apoyo en la edición y revisión crítica de este manuscrito.

\section{Referencias}

1 Tsukiboshi M, Andreasen JO, Asai Y, Bakland LK, Wilson TG. Clasification and clinical indications. Autotransplantation of Teeth. Chicago: Quintessence Publishing Co, Inc; 2001. pp 57-74.

2 Ahn S, Jang J, Seo J, Cho KM, Jung SH, Lee HW et al. Influence of cryopreservation methods to induce CCL-13 from dental pulp cells. J Endod. 2013; 39 (12): pp 15621566.

3 Hata G, Yoshikawa S, Toda T. Autotransplantation using endosseous implants as stabilizers. J Endod. 1991; 17 (3): pp 127-130.

4 Ballinas J, Ramos P, Rodríguez F, Jácome A. Autotrasplante de un tercer molar inmaduro: reporte de caso. Revista ADM 2017; 74 (2): pp 100-106

5 Bae JH, Choi YH, Cho BH, Kim YK, Kim SG. Autotransplantation of teeth with complete root formation: a case series. J Endod. 2010; 36 (8): pp 1422-1426.

6 Kim S, Lee S, Shin Y, Kim E. Vertical bone growth after transplantation of mature third Molars: 2 case reports with long-term follow-up. J Endod. 2015; 41 (8): pp 1371-1374.

7 Filipe L, Bochnia B, Brasil F, Maruo H, Guariza FO, Motohiro TO. Root formation of an autotransplanted tooth. Case report. Dent Traumatol. 2009; 25: pp 341-345.

8 Smith JJ, Wayman BE. Succesful autotransplantation. J Endod. 1987; 13 (2): 77-80. 
9 Teixeira CS, Pasternak Jr, Vansan LP, Sousa-Neto MD. Autogenous transplantation of teeth with complete root formation: two case reports. Int Endod J. 2006; 39: pp 977-985.

10 Mejía JL, Marcano M, Vera J. Autotransplantation of premolar: a long-term follow-up report of a clinic case. J 22 Endod. 2012; 38 (8): 1149-1152.

11 Laureys WG, Cuvelier CA, Dermaut LR, De Pauw GA. The critical apical diameter to obtain regeneration of the 23 pulp tissue after tooth transplantation, replantation, or regenerative endodontic treatment. J Endod. 2013; 39 (6): pp 759-763.

12 Intra JB, Roldi A, Brandão RC, de Araújo EC, Estrela C. 24 Autogenous premolar transplantation into artificial socket in maxillary lateral incisor site. J Endod. 2014; 40 (11): pp $1885-1890$

13 Lim JH, Huh JK, Park KH, Shin SJ. Autotransplantation of an impacted premolar using collagen sponge after cyst enucleation. J Endod. 2015; 41 (3): 417-419.

14 Park YS, Baek SH, Lee WC, Kum KY, Shon WJ. Autotransplantation with simultaneous sinus floor elevation. J Endod. 2012; 38 (1): pp 121-124.

15 Bauss O, Schilke R, Fenske C, Engelke W, Kiliaridis S. Autotransplantation of immature third molars: influence of different splinting methods and fixation periods. Dent Traumatol. 2002; 18: pp 322-328

16 Aparicio P, Basili A, Martínez B. Autotrasplante de gérmenes de terceros molares: estudio prospectivo del post- operatorio en ocho pacientes. Acta Odontológica Venezolana. 2009; 47(3): p 3.

17 Puigdollers A. Cirugía auxiliar, cirugía correctiva. Rev Esp Ortod. 2002; 32: p 266.

18 Ghassemi M, Jaimilian A, Fritz U, Riediger D, Ghassemi A. Orthodontic treatment after autotransplantation. Angle Orthod. 2011; 81(4):pp 721-725.

19 Consolaro A. Novaes T, Batista J, Roldi A. Transplantes dentários autógenos: uma solução para casos ortodônticos e uma casuística brasileira. Rev Dent Press Ortodon Ortop Facial. 2008; 13 (2):pp 23-8.

20 Nahimura K, Amano S, Nakao K, Goto S. Orthdontic treatment including autotransplantation of a mature tooth. Angle Orthod. 2009; 79 (2): pp 387-93.
21 Paulsen H, Andreasen J, Schwartz O. Pulp and periodontal healing, root development and root resorption subsequent to transplantation and orthodontic rotation: A long term study of autotransplanted premolars. Am J Orthod Dentofac Orthop. 1995; 108:pp 630-40.

22 Lim W, Chun Y. Orthodontic treatment combined with autotransplantation after removal of ameloblastoma. Am J Orthod Dentofacial Orthop. 2009; 135:pp 375-379.

$23 \mathrm{Ru} \mathrm{N}$, Yuxing B. Canine autotransplantation: Effect of extraction site preservation with a titanium prosthesis and a bioresorbable membrane. Am J Orthod Dentofac. 2013; 143 (5): pp 724-734.

24 Azevedo P, Gomes C, Zanetta-Barbosa D, Barmadineli N, Gerais M. Oral Surg Oral Med Oral Pathol Oral Radiol Endond. 2007; 104:pp 287-93.

25 Jonsson T, Sigurdsson T. Autotransplantation of premolars to premolar sites. A long-term follow-up study of 40 cosecutive patients. Am J Orthod Dentofacial Orthop. 2004; 125: pp 668-675.

Recibido: 16 de Julio de 2018.

Aceptado: 30 de Octubre de 2019. 